\title{
The origin of the strong soft excess and puzzling iron line complex in Mkn 841
}

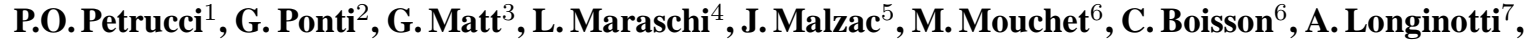 \\ K. Nandra ${ }^{8}$, P. Ferrando ${ }^{9}$, and G. Henri ${ }^{1}$ \\ 1 Laboratoire d'Astrophysique de Grenoble, BP 43, 38041 Grenoble Cedex 9, France \\ 2 NAF-IASF Sezione di Bologna, Via Gobetti 101, I-40129 Bologna, Italy \\ 3 Dipartimento di Fisica, Università degli Studi "Roma tre", via della Vasca Navale 84, I-00046 Roma, Italy \\ 4 Osservatorio Astronomico di Brera, Via Brera 28, 02121 Milano, Italy \\ 5 Centre d'étude Spatiale des Rayonnements (CNRS/UPS/OMP), 31028 Toulouse, France \\ 6 LUTH, Observatoire de Paris, Section de Meudon, 92195 Meudon Cedex, France \\ 7 XMM-Newton Science Operations Center, European Space Astronomy Center, ESA, 28080 Madrid, Spain \\ 8 Astrophysics Group, Imperial College London, Blackett Laboratory, Prince Consort Road, London SW7 2AW \\ ${ }^{9}$ Service d'Astrophysique, DSM/DAPNIA/SAp, CE Saclay, 91191 Gif-sur-Yvette Cedex, France
}

Received 30 May 2005, accepted 11 Nov 2005

Published online later

Key words galaxies: individual (Mkn 841) - galaxies: Seyfert - X-rays: galaxies

Mkn 841 has been observed during 3 different periods (January 2001, January 2005 and July 2005) by XMM-Newton for a total cumulated exposure time of $\sim 108 \mathrm{ks}$. We present in this paper a broad band spectral analysis of the complete EPIC-pn data sets. These observations confirm the presence of the strong soft excess and complex iron line profile known to be present in this source since a long time. They also reveal their extreme and puzzling spectral and temporal behaviors. Indeed, the $0.5-2 \mathrm{keV}$ soft X-ray flux decreases by a factor 3 between 2001 and 2005 and the line shape appears to be a mixed of broad and narrow components, both variable but on different timescales. The broad-band 0.5-10 keV spectra are well described by a model including a primary power law continuum, a blurred photoionized reflection and a narrow iron line, the blurred reflection fitting self-consistently the soft excess and the broad line component. The origin and nature of the narrow component is unclear.

(c) 2006 WILEY-VCH Verlag GmbH \& Co. KGaA, Weinheim

\section{Introduction}

Mkn 841 is a bright Seyfert 1 galaxy ( $\mathrm{z}=0.0364$, Falco et al. 2000), one of the rare Seyfert 1 detected by OSSE at more than $3 \sigma$ (Johnson 1997). It is known for its large spectral variability (George et al. 1993; Nandra et al. 1995), its strong soft excess (this was the first object where a soft excess was observed, Arnaud et al. 1985) and its variable iron line (at least on a year time scale, George et al. 1993). Here we report the data analysis of the complete set of XMMNewton observations of this source, focusing on the EPICpn instrument. These observations confirm the presence of the soft excess and iron line complex and reveal their extreme and puzzling spectral and temporal behaviors.

\section{Data}

Mkn 841 has been observed 5 times during 3 different periods (January 2001, January 2005 and July 2005) by XMMNewton for a total cumulated exposure time of $\sim 108 \mathrm{ks}$. Table 1 gives a summary of the different XMM-Newton pointings with the corresponding dates, exposures and count rates. For the spectral analysis we have divided OBS4 (jan.
2005) in 3 parts (noted part1, part2 and part3 in the following) of about $15 \mathrm{ks}$ each.

The EPIC-pn camera was always operated in Small Win-I dow mode, with thin aluminium filters to reject visible light. The EPIC-pn event files were reprocessed from the ODF data files using the epchain pipeline tasks of the XMM Science Analysis System (SAS version 6.5) and using the most updated version of the public calibration files. These event files were then filtered for good time intervals following the "recipe" given in the XMM handbook (v2.01 23 July 2004, Sect. 5.2.4). The source spectra and light curves were built from photons detected within a 40 arcsec extraction window centered on the source. X-ray events corresponding to patterns $\leq 4$ were selected. The background was estimated within a window of the same size from an offset position.

In the following, all errors refer to $90 \%$ confidence level for 1 interesting parameter $\left(\Delta \chi^{2}=2.7\right)$.

\section{Light curves, hardness ratios}

We have plotted on top of Fig. 1 the $0.5-10 \mathrm{keV}$ EPIC-pn count rate light curves of the different XMM-Newton observations of Mkn 841 as well as the hardness ratio (5-10)/(35 ) at the bottom. The time binning is $500 \mathrm{sec}$. The $0.5-10$ 
Table 1 Observation epochs, exposure and mean count rates.

\begin{tabular}{lccccc}
\hline & OBS 1 & OBS 2 & OBS 3 & OBS4 & OBS5 \\
\hline Start date & $2001-01-13$ & $2001-01-13$ & $2001-01-14$ & $2005-01-16$ & $2005-07-17$ \\
& $(05 \mathrm{~h} 20 \mathrm{~m} 55 \mathrm{~s} \mathrm{UT})$ & $(09 \mathrm{~h} 33 \mathrm{~m} 50 \mathrm{~s} \mathrm{UT})$ & $(00 \mathrm{~h} 52 \mathrm{~m} 28 \mathrm{~s} \mathrm{UT})$ & $(12 \mathrm{~h} 38 \mathrm{~m} 21 \mathrm{~s} \mathrm{UT})$ & $(06 \mathrm{~h} 38 \mathrm{~m} 03 \mathrm{~s} \mathrm{UT})$ \\
Exposure (s) & 8449 & 10900 & 13360 & 45982 & 29071 \\
Cts.s ${ }^{-1} \mathrm{PN}$ & 18.0 & 22.2 & 21.8 & 5.6 & 7.2 \\
\hline
\end{tabular}

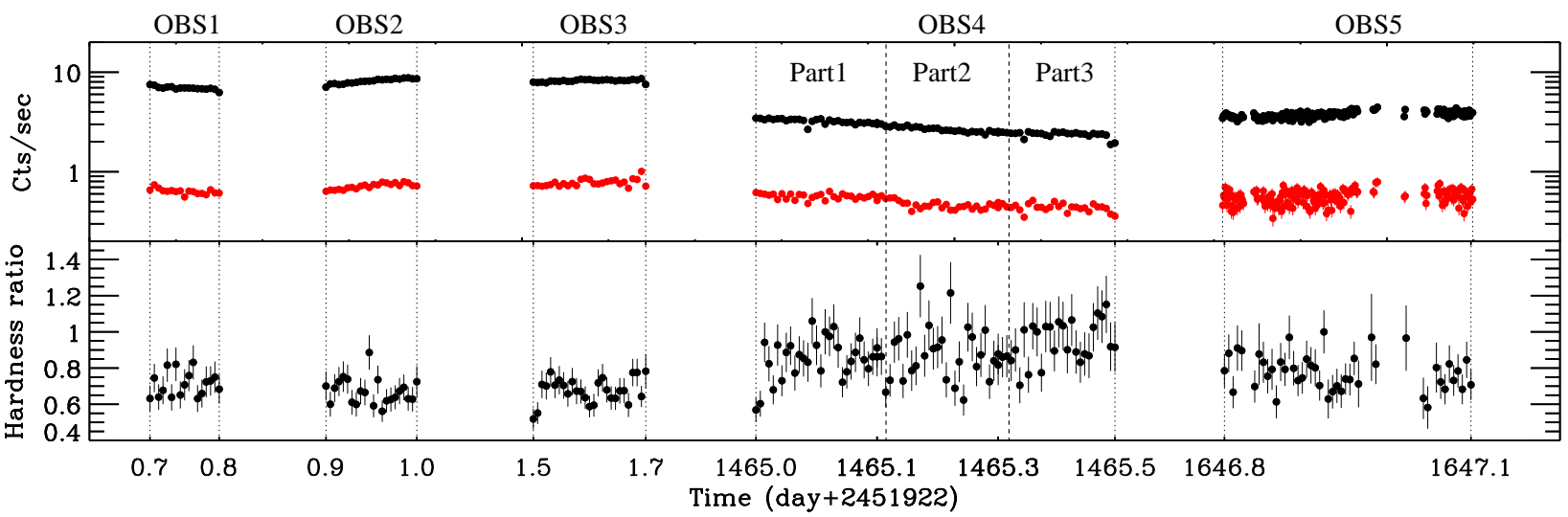

Fig. 1 Upper panel: Total (0.5-10 keV, upper curve) and hard (3-10 keV, lower curve) X-ray light curves of the different EPIC-pn observations of Mkn 841. The broad band flux is clearly dominated by the soft $(<3 \mathrm{keV})$ bands. The soft X-rays count rate varies by a factor 4 in 4 years while the hard X-ray one varies by only $\sim 60 \%$. On the other hand flux variability up to $\sim 50 \%$ is also observed on tens of ks. The hardness ratio is plotted on the lower panel.

$\mathrm{keV}$ count rate decreases by a factor $\sim 4$ in 4 years while the hardness ratio increases, reaching maximum values during OBS 4. The 3-10 keV count rate has been also plotted in Fig. 11 It shows variations of $\sim 60 \%$ implying that the $0.5-$ $10 \mathrm{keV}$ count rate variability is dominated by the soft $(<3$ $\mathrm{keV}) \mathrm{X}$-ray variability, at least on long time scale. Smooth soft and hard flux variabilities up to $\sim 50 \%$ are also visible on tens of ks.

\section{Simple spectral analysis}

We use first very simple spectral components to reproduce the observed features. We use a power law for the continuum and a gaussian for the iron line. We fit the data above 3 $\mathrm{keV}$ first. Then we fix the different parameters and include the data below $3 \mathrm{keV}$ down to $0.5 \mathrm{keV}$ and we add a simple black body component to model the soft excess. The best fits are not satisfactory for all the observations due to discrepancies present below $3 \mathrm{keV}$. Anyway different remarks, weakly affected by the goodness of the fit, can already been done:

1) The spectral variability appears to be due to both variable high energy (above $3 \mathrm{keV}$ ) continuum and variable soft excess. Indeed the photon index varies by $\sim 0.5$ between 2001 and 2005, while the soft X-ray flux, below $3 \mathrm{keV}$, decreases by a factor 3 , i.e. more than the flux variability (of $\sim 60 \%$ ) expected if the power law continuum was the only variable component.

2) The photon index reaches values as small as 1.3 during OBS 4, unusually hard for a Seyfert X-ray spectrum.

3) The best fit requires a narrow line in all the observations but OBS 3 and part 2 of OSB 4 where a broad com-
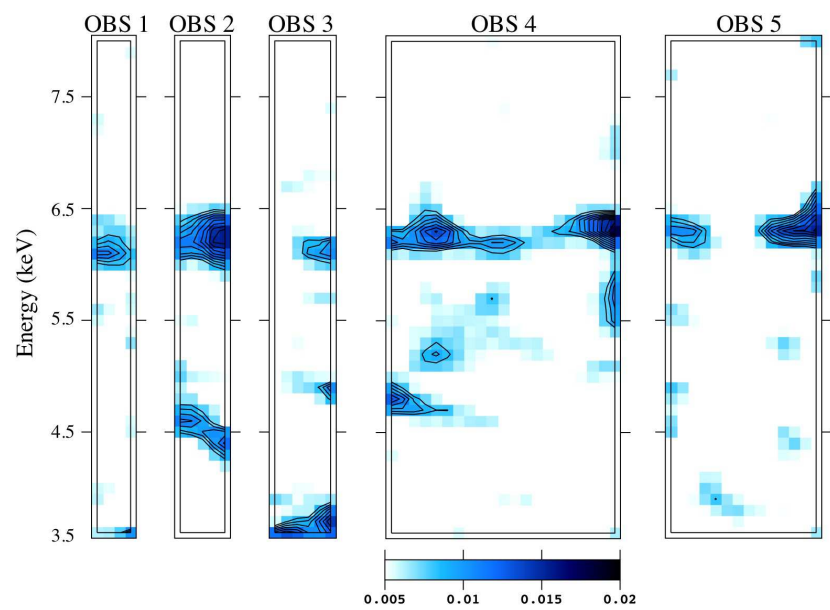

Fig. 2 Map excess of the different XMM observations

ponent is found. The apparent variability of the line width on short time scale in 2001 was already noted by Petrucci et al. (2002) and Longinotti et al. (2004) and we observe the same variability behavior during the observation of Jan. 2005. This variability is clearly visible on the map excess reported in Fig. 2 (and produced following the method describe in Iwasawa et al. 2004). The contour plots, at 68 and $90 \%$ confidence level, of the line flux vs. line width for the different observations are plotted in Fig. 3 .

Somes differences exist however between the observations of Jan. 2001 and Jan. 2005. First of all, we note an increase of the line flux during part 2 of OBS4, a factor 2-3 larger than in part 1 and 3 while in 2001 the line flux kept constant between the narrow and broad component. More importantly we detect some residuals of a narrow compo- 

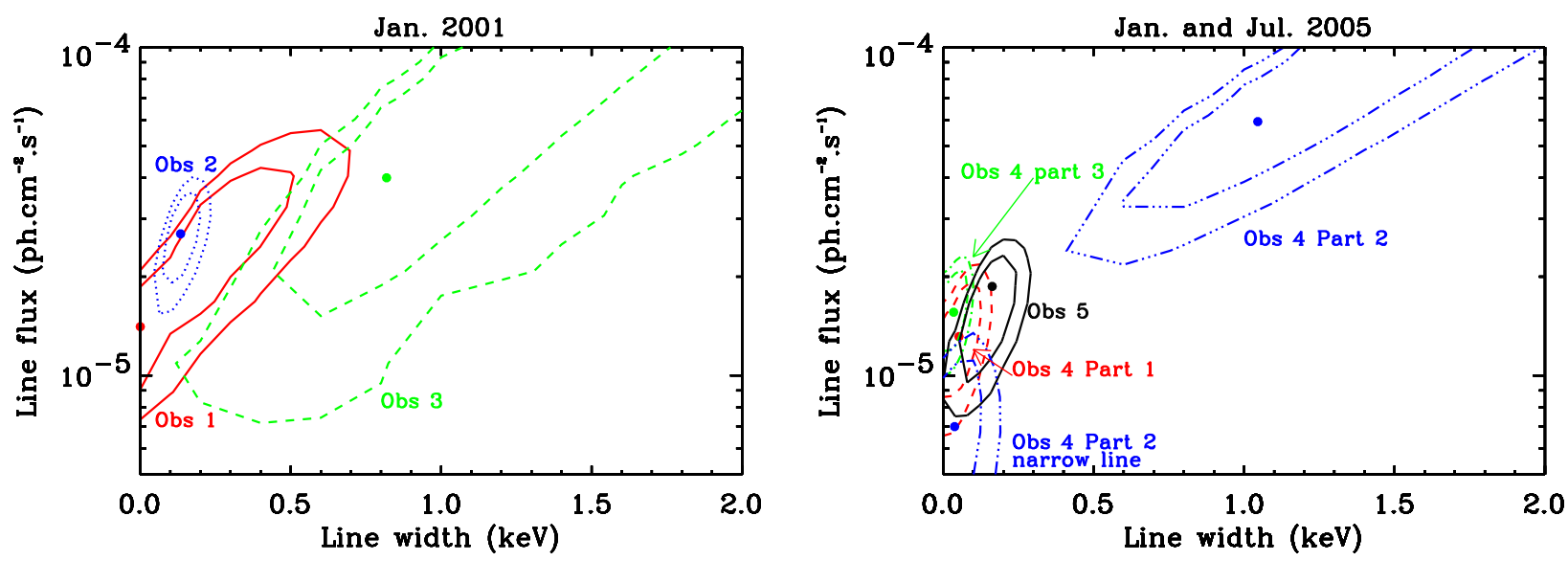

Fig. 3 Contour plots (68 and $90 \%$ ) of the line width vs. line flux for the 3 observations of Jan 2001 (left) and the 3 parts of Jan. 2005 (right). In both cases, the model includes a simple power law and a gaussian line. We have also over plotted on the right the contour plots of OSB 5 as well as the contours of the narrow component detected in part 2 of OBS 4 .

nent near $6.4 \mathrm{keV}$ in part 2 of OBS 4 . There are also some residuals near 5-6 keV in part 1 and 3 suggesting the presence, in these cases, of a broad component. To check this, we have added a second gaussian to the model. A narrow component is indeed detected in part 2 while broad ones are found in part 1 and 3 . The addition of this second line is significant at more than $99.7 \%, 93 \%$ and $98.9 \%$ (following the F test) for part 1, 2 and 3 respectively. Thus the line complex during OBS 4 appears to be a mix of broad and narrow components. This is also illustrated in Fig. 4 where we have plotted the ratio of the complete OBS 4 data spectrum and the best fit power law obtained fitting only the data above 3 $\mathrm{keV}$ but ignoring the 4-7 keV energy range. The presence of both broad and narrow components is clearly visible. This contrasts with what we observed in the other observations (OBS 1, 2 or 3 and OBS 5) where the addition of a second gaussian line does not improve significantly the fits.

It is also worth noting that the narrow line detected in part 2 of OBS 4 has a weak flux but in relatively good agreement with the fluxes of the other narrow components detected in OBS 1 and OBS 2 in 2001, in part 1 and 3 of OBS 4 and in OBS 5 . The corresponding contour plot has been over-plotted on Fig. 3 This would suggest the narrow line to be produced in a remote material but the lack of a narrow component in OBS 3 disagrees with this picture. Thus the origin of this component is unclear. Concerning the broad components detected in part 1 and 3, they are consistent with each other and with the broad component of part 2 .

We have also tried to fit the 3 parts of OBS 4 with a model of line emission from a relativistic accretion disk (DISKLINE model of XSPEC) fixing the accretion disc outer radius to $1000 r_{g}$ and keeping the inclination angle constant between the different spectra. The fit is good, with a best fit inclination angle of $47_{-8}^{+5}$ degrees. We note however that the fit with two gaussians is slightly better for part 3 of OBS 4 compared to the DISKLINE one $\left(\chi^{2} /\right.$ dof $f=131 / 140$ compared to $141 / 141$ ) thanks to a better fit of the narrow component.

\section{A more physical analysis: blurred ionized reflection?}

Recent studies suggest that an appealing explanation for the presence of strong soft excesses in AGNs could be blurred (photoionized) reflection from the accretion disc (e.g. Crummy et al. 2005). These authors already applied this model to the OBS 3 of Mkn 841 with success. Moreover, in the cases in which a broad Fe line is clearly detected (such as in MCG-6-30-15) the model is very robust because the soft excess and broad Fe line are fitted self-consistently with the same relativistically blurred reflection model. The characteristics of the line complex in Mkn 841, with the presence of a variable broad line component well fitted by a blurred (DISKLINE) profile, and a strong soft excess, suggest that photoionized reflection may play also an important role in this source.

To test these assumptions we have used a more physical model with the following components: 1) a neutral absorption free to vary above the galactic value 2) a cut-off

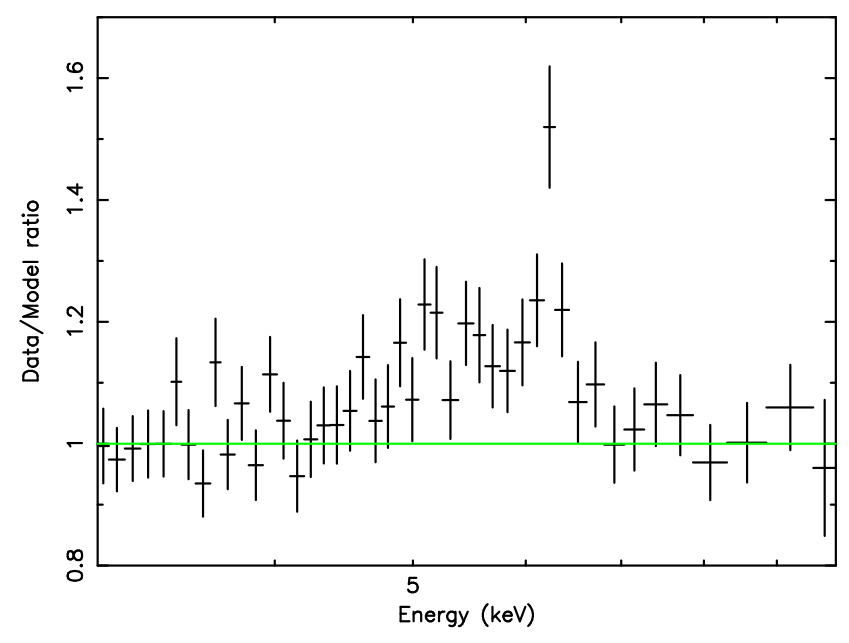

Fig. 4 Ratio data/model for OBS 4. The model is a simple power law fitted between 3 and $10 \mathrm{keV}$, ignoring the $4-7 \mathrm{keV}$ range. Broad and narrow components are clearly visible. 
P.O. Petrucci et al.: Soft excess and Iron line complex in Mkn 841

Table 2 Best fit results obtained with the blurred ionized reflection model.

\begin{tabular}{lccccc}
\hline Obs. & $\Gamma$ & $\xi$ & $q$ & $\begin{array}{c}r_{i n} \\
r_{g}\end{array}$ & $\chi^{2} /$ dof \\
\hline 1 & $2.31_{-0.09}^{+0.07}$ & $190_{-80}^{+160}$ & $8.7_{-1.4}^{+1.3}$ & $1.3_{-0.1}^{+0.2}$ & $248 / 241$ \\
2 & $2.28_{-0.05}^{+0.05}$ & $117_{-10}^{+140}$ & $8.2_{-1.2}^{+1.6}$ & $1.4_{-0.2}^{+0.1}$ & $227 / 260$ \\
3 & $2.19_{-0.04}^{+0.08}$ & $54_{-12}^{+54}$ & $5.3_{-0.7}^{+3.1}$ & $1.4_{-0.2}^{+0.2}$ & $292 / 273$ \\
$4-1$ & $1.50_{-0.02}^{+0.10}$ & $200_{-20}^{+10}$ & $6.4_{-0.5}^{+0.6}$ & $1.8_{-0.6}^{+0.2}$ & $283 / 269$ \\
$4-2$ & $1.57_{-0.04}^{+0.05}$ & $140_{-7}^{+2}$ & $6.0_{-0.3}^{+0.3}$ & $1.7_{-0.1}^{+0.1}$ & $292 / 264$ \\
$4-3$ & $1.49_{-0.04}^{+0.02}$ & $140_{-10}^{+60}$ & $7.0_{-0.7}^{+1.1}$ & $2.1_{-0.2}^{+0.1}$ & $283 / 264$ \\
5 & $1.63_{-0.02}^{+0.01}$ & $300_{-40}^{+10}$ & $7.0_{-0.6}^{+1.2}$ & $2.0_{-0.1}^{+0.2}$ & $371 / 278$ \\
\hline
\end{tabular}

power law continuum (the high energy cut-off being fixed to $300 \mathrm{keV}$ ), 3) a blurred photoionized reflection and 4) a narrow line component if needed. For the blurred reflection, we use the tables of the Ross \& Fabian code (Ross \& Fabian 2005 ) convolved with a Laor profile (KDBLUR kernel). This component is expected to reproduce the soft excess and the broad iron line. The inner radius of the reflecting accretion disc as well as the disc emissivity law spectra index were let free to vary in the Laor kernel. The outer radius was fixed to 400 Schwarschild radii. The iron abundance was also fixed to the solar one for the computation of the ionized reflection. To constrain the inclination angle, a first step was to fit all the data sets simultaneously keeping the inclination angle constant between each observation. We obtain a best fit with an inclination angle of $\sim 50 \mathrm{deg}$. in agreement with the best fit angle obtained with the DISKLINE model in the previous section (and also close to the inclination angle found by Crummy et al. 2005 for OBS 3). The use of the complete set of data was however too time consuming for the computation of the errors. Thus, a second step was to study

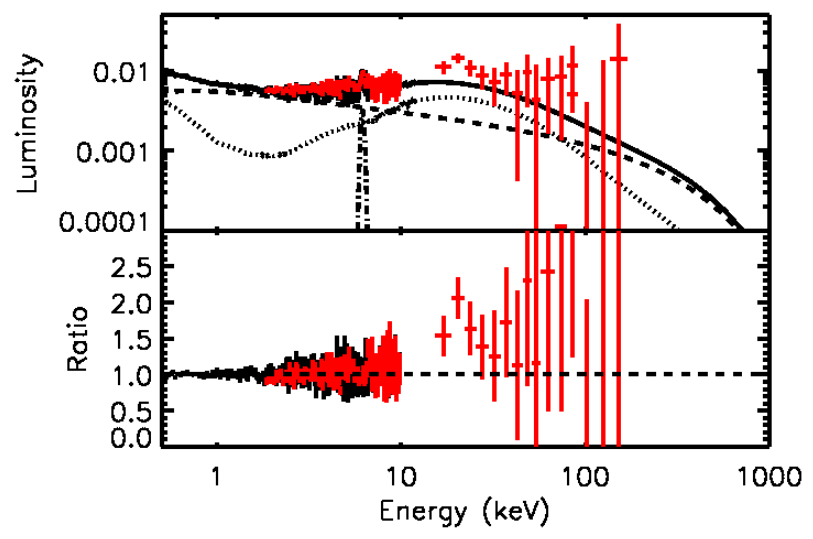

Fig. 5 Top: The solid line is the best fit blurred ionized reflection model of OBS 1, the cut-off power law continuum and blurred ionized reflection being plotted in dashed and dotted lines respectively. The dot-dashed line is the narrow iron line. The dark (black) crosses are the OBS 1 XMM data and the clear (red) crosses are the MECS and PDS data of the simultaneous $100 \mathrm{ks}$ BeppoSAX. The PDS/MECS cross normalization was fixed and only the XMM-pn/MECS one was let free to vary. Bottom: Data/model ratio the different observations separatly but fixing the inclination angle to the previous value.

We obtain very good fits in most of the cases, with some problems with OBS 5 where important absorption features are present at low energies (the fit is highly improved $\left(\Delta \chi^{2}\right.$ $=81$ ) with the addition of a warm absorber component ABSORI in XSPEC). This model depends strongly on the emissivity law index $q\left(j \propto r^{-q}\right)$ and the inner disc radius $r_{i n}$. Thus the fit of the broad line component as well as the soft excess requires a fine tuning of these two parameters. For example, large $q$ and small $r_{i n}$ imply strong relativistic effects that strongly smeared the lines. This is indeed what we find in the case of OBS 1, OBS 2 and OBS 5 where no broad line is detected (cf. Table 2. On the other hand, for a given $r_{i n}$, smaller $q$ reduces the importance of the relativistic blurring effects and the broad line is more easily detectable like in OBS 3 and OBS 4. The smaller values of $q$ are obtained for OBS 3 and part 2 of OBS 4 where the stronger broad lines are detected.

It is worth noting that in almost all cases but OBS 3 the addition of a narrow line is required by the data. This is due to the large blurring effects needed to fit the soft excess that smeared the iron line and then prevent a good fit of the narrow component. With this model the soft band variability is due to both blurred reflection and continuum variability while the hard band (above $3 \mathrm{keV}$ ) is dominated by the power law continuum. This model predicts however large reflection component above $10 \mathrm{keV}$ i.e. outside the XMM energy range. In the case of the 2001 observations, we have $100 \mathrm{ks}$ of simultaneous BeppoSAX observation, the BeppoSAX observation window including OBS 1, OBS 2 and OBS 3. The BeppoSAX data show quite good agreements with the XMM best fit models obtained in 2001 (cf. Fig. 5) which is quite encouraging.

\section{Conclusion}

The present analysis of the complete set of XMM/EPIC-pn data of Mkn 841 reveals extreme and puzzling spectral and temporal behaviors of its soft excess and iron line complex. If the soft excess and broad iron line can be well fitted by an ionized blurred reflection model, the origin of the narrow line component is still not understood. Future Suzaku observations $(2 \times 50 \mathrm{ks})$ will be of crucial help to conclude on this source.

\section{References}

Arnaud, K., Branduardi-Raymont, G., Culhane, J., et al.: 1985, MNRAS 217, 105

Crummy J., Fabian, A., Brandt, W., Boller, Th.: 2006, MNRAS 365,1067

Falco, E., Kurtz, M., Geller, M., et al.: 1999, PASP 111, 438

George, I., Nandra, K., Fabian, A., et al. 1993: MNRAS 260, 111

Iwasawa, K., Miniutti, G., Fabian, A.: 2004, MNRAS 355, 1073

Johnson, N.: 1997, BAAS 190, 2005

Longinotti, A., Nandra, K., Petrucci, P., O’Neill, P.: 2004, MNRAS 355, 929

Nandra, K., Turner, T., George, I., et al.: 1995, MNRAS 273, 85 
Petrucci, P., Henri, G., Maraschi, L., et al.: 2002, A\&A 388, L5

Ross R., \& Fabian, A.: 2005, MNRAS 358, 211 